\title{
Possible Lattice Distortions in the Hubbard Model for Graphen€
}

\author{
Rupert L. Frank ${ }^{1,}$, and Elliott H. Lieb ${ }^{1,2}$, 国 \\ ${ }^{1}$ Department of Mathematics, Princeton University, Washington Road, Princeton, NJ 08544, USA \\ ${ }^{2}$ Department of Physics, Princeton University, P. O. Box 708, Princeton, NJ 08542, USA
}

\begin{abstract}
The Hubbard model on the honeycomb lattice is a well known model for graphene. Equally well known is the Peierls type of instability of the lattice bond lengths. In the context of these two approximations we ask and answer the question of the possible lattice distortions for graphene in zero magnetic field. The answer is that in the thermodynamic limit only periodic, reflectionsymmetric distortions are allowed and these have at most 6 atoms per unit cell as compared to two atoms for the undistorted lattice.
\end{abstract}

PACS numbers: 71.10.Fd, 61.48.Gh, 73.22.Pr, 05.30.Fk

Graphene is a two-dimensional array of carbon atoms arranged in a honeycomb lattice. The number of delocalized electrons, $N$, equals the number of carbon atoms, i.e., the conduction band is half-filled. The dynamics of the electrons is often modelled by the Hubbard Hamiltonian

$$
\begin{aligned}
H(T)= & -\sum_{\sigma=\uparrow, \downarrow} \sum_{\langle x, y\rangle} t_{x y}\left(c_{x, \sigma}^{\dagger} c_{y, \sigma}+\text { h.c. }\right) \\
& +U \sum_{x}\left(n_{x \uparrow}-\frac{1}{2}\right)\left(n_{x \downarrow}-\frac{1}{2}\right)+\sum_{\langle x, y\rangle} F\left(t_{x y}\right) .
\end{aligned}
$$

The $c_{x, \sigma}$ are fermion annihilation operators and $n_{x, \sigma}=$ $c_{x, \sigma}^{\dagger} c_{x, \sigma}$. For the purposes of this paper the on-site repulsion $U$ can have any sign as long as it is the same for all lattice sites. In particular, for $U=0$ we have the Hückel model.

The notation emphasizes the dependence on the nearest-neighbor hopping matrix $T=\left(t_{x y}\right)$. We take the hopping matrix elements $t_{x y}$ to be positive, although not necessarily independent of the pair $x, y$. (By the wellknown particle-hole transformation on a bipartite lattice, we could, as well, take the $t_{x y}$ 's negative.) It is important for us that $t_{x y}$ is real, i.e., that there is no magnetic field.

The interesting quantity, which does not usually appear in the Hubbard model, is the distortion energy $F\left(t_{x y}\right)$. What we are assuming is that $t_{x y}$ depends on the physical distance $d_{x y}$ between the lattice sites $x$ and $y$. There is an equilibrium value $d^{(0)}$ and deviations from this value cost a positive energy. After eliminating $d_{x y}$ we can assume that the distortion energy depends on $t_{x y}$. This is the quantity $F\left(t_{x y}\right)$ in (11). Thus, $F(t)$ has a minimum at $t=t^{(0)}$, corresponding to $d^{(0)}$, and it goes to infinity as $t$ goes to zero (infinitely separated atoms) or to infinity (no atomic separation). We do not have to assume that $F$ is convex. This model, while quite general, does not take account of the possible distortion energy connected with the change in bond angles or possible non-planar atomic configurations, i.e., curvature of the lattice. It also does not take account of longer range interactions [1, 2].

The problem we address is the possible configurations

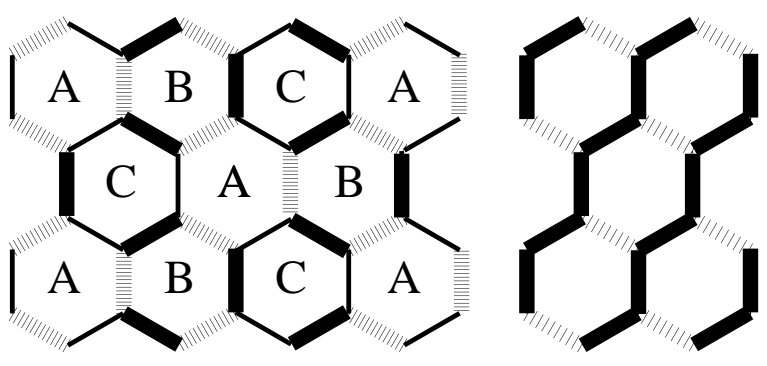

FIG. 1: (a) On the left is the only graphene structure allowed by our theorem. The locations of the three possible bond lengths are indicated by heavy-solid, light-solid and dashed lines. There are six atoms (or three hexagons) per unit cell. The Kekule structure is the special case of equal length solid lines. (b) The ALT structure on the right breaks some of the reflection symmetry and is not allowed by our theorem.

$T=\left(t_{x y}\right)$ that minimize the ground state energy $E(T)$ of $H(T)$. We answer this in the thermodynamic limit by proving that one ends up with a relatively simple periodic configuration. Nothing more complicated can occur! This periodic configuration is shown in Fig. 1(a), where it will be seen that the resulting lattice can have at most three different lengths and the unit cell can have at most six atoms. A special case is the so-called Kekulé lattice in which two of the lengths are equal. It also has six atoms per unit cell. Many authors have considered various possible energy-minimizing periodic distortions. The discussion can and should also be extended to include non-periodic and chaotic structures. Our contribution is to rigorously exclude anything more complicated than that shown in Fig. 1(a), in the thermodynamic limit. In particular, the ALT structure of Ref. [3 5] in Fig. 1(b) is not an energy-minimizer for the Hubbard model, since it breaks two of the three reflection symmetries shown in Fig. 1(a).

The idea that distortion of a lattice can lower the energy is sometimes called the Peierls instability, but the idea is older (see [6]). Peierls' essential contribution [7] was to realize that this distortion always occurs in 1D for an infinitely long chain or ring of atoms. It does not 
always occur in higher dimensions, or in a finite 1D ring, e.g., benzene.

Let us recall the situation in one dimension, i.e., the annulenes like benzene or polyacetylene. It has been proved [6, 8] that for a closed ring of $N$ atoms, with Hamiltonian as in (11), the minimum energy configuration is precisely the one in which there are two alternating bond lengths $l_{1}$ and $l_{2}$, provided $N=2 \bmod 4(N=6,10,14, \ldots)$. It is possible that $l_{1}=l_{2}$, as is the case in benzene, but nothing more complicated than dimerization can ever occur in this model, for any $F$-function. When $N=0 \mathrm{mod}$ 4 more complicated things can occur when $F$ is not a quadratic, e.g., for $N=4$ the minimum configuration can be a trapezoid [8]. Nevertheless, as $N$ goes to infinity the dimerized energy is always asymptotically exact. The general case is analyzed in [6] in detail and the stated conclusion is even shown to apply to the so-called 'SpinPeierls model'.

The method of proof for 1D does not generally extend to higher dimensions. It does not extend to the square lattice unless a physically unreachable magnetic field is imposed [9], but, as we discover here, it does extend to the hexagonal lattice with zero magnetic field. The reason for this difference, ultimately, is that the energy minimizing magnetic flux for the square lattice is non-zero, whereas for the hexagonal lattice it is zero, as assumed in $H(T)$ above [9].

Naturally, we cannot expect perfect periodicity in a finite lattice, because there will always be edge effects. This remark motivates the following theorem, whose proof we will outline here.

Theorem. - For any triplet of hopping matrix elements $\left(t^{(1)}, t^{(2)}, t^{(3)}\right)$ let $e\left(t^{(1)}, t^{(2)}, t^{(3)}\right)$ denote the ground state energy per atom in the thermodynamic limit for the Hubbard model (1D) with these t's arranged as in Fig. 1(a). Let $e$ be the minumum of $e\left(t^{(1)}, t^{(2)}, t^{(3)}\right)$ with respect to these $t$ 's. Then the ground state energy $E(T)$ of (11) with arbitrary t's on a large, finite lattice $\Lambda$, with $|\Lambda|$ sites, satisfies

$$
E(T) \geq|\Lambda| e+o(|\Lambda|),
$$

where $o(|\Lambda|) \leq$ const $|\Lambda|^{1 / 2} \ln |\Lambda|$. The same conclusion holds for the free energy at finite temperature.

Strictly speaking, we prove that there is a minimizer (to leading order) with the structure of Fig. 1(a), but we do not rigorously prove uniqueness of this structure. Nevertheless the uniqueness is evident for two reasons: One is that our proof proceeds by showing that repeated reflection of a single line cannot raise the energy, but in reality it surely lowers it. The second is that the structure made this way out of repeated reflections still has the hopping matrix elements that were in the given row before it was reflected. At this point we could lower the energy still further by reevaluating the optimum $t$-values. These will surely change, and the energy will be lower because the equation for the optimum values, namely,
$F^{\prime}\left(t_{x y}\right)-2\left\langle c_{x}^{\dagger} c_{y}\right\rangle=d E / d t_{x y}=0$ will likely no longer be satisfied with the old $t$ values. For example, the ALT structure in Fig. 1(b) morphs into the Fig. 1(a) structure of the Kekule type. If the $t$ value on the heavy bond did not change, then the expectation value of $c_{x}^{\dagger} c_{y}$ along the distinguished bond would have to be the same as for the undistinguished bond - which it is not.

We recall two facts about the Hubbard model (11), which hold even for arbitrary $T$ on any finite, bipartite lattice $\Lambda$ with an even number of sites.

(1) Among the absolute ground states there is one with $N=|\Lambda|=$ number of sites. If $U \neq 0$, this state is unique [6]. This means that we do not have to worry about constraining the particle number to be equal to the number of lattice sites, $|\Lambda|$. This constraint is automatic.

(2) Among the ground states there is one with total spin zero [10].

The main tool we are going to use is reflection positivity which originated in quantum field theory [11. It was later used in statistical mechanics [12 16 to prove long range order of systems with continuous symmetry. It was also important for static situations like the solution of the flux phase problem [9]. It is in the latter sense that we apply it here. Our theorem above can be rephrased by saying that the lowest energy occurs for a lattice configuration that preserves the three-fold reflection symmetry of the hexagonal lattice, as shown in Fig. 1(a).

Let $\Lambda$ be piece of a hexagonal lattice that is reflection symmetric about a line $l$ that passes perpendicularly through bonds. The two halves of the lattice will be called $\Lambda_{L}$ and $\Lambda_{R}$. Let $T_{L}$ and $T_{R}$ denote the hopping elements on the left and on the right side, respectively, and let $T_{M}$ be those corresponding to bonds cut by $l$. Let $T_{L}^{*}$ be the reflection of $T_{L}$ to the right and, similarly, let $T_{R}^{*}$ be the reflection of $T_{R}$ to the left. Then, symbolically, $T=\left(T_{L}, T_{M}, T_{R}\right)$ and we want to consider $T^{L}=\left(T_{L}, T_{M}, T_{L}^{*}\right)$ and $T^{R}=\left(T_{R}^{*}, T_{M}, T_{R}\right)$. The corresponding ground state energies are related by the basic inequality, whose proof is in [6, 9,17$]$;

$$
E(T) \geq \frac{1}{2} E\left(T^{L}\right)+\frac{1}{2} E\left(T^{R}\right) .
$$

Our way forward is now obvious. Think of an infinite hexagonal lattice. There are three directions in space for which we can draw lines in these directions that cut lattice bonds perpendicularly (but do not pass through sites). For each of these three directions there are infinitely many parallel lines with these property. We would like to use reflection positivity for each of them to say that if the hopping matrix elements are not as shown in Fig. 1(a), then we can lower the energy by reflecting one half of the lattice onto the other half and keeping the better of the two choices according to inequality (3). In short, if the optimum configuration did not have the symmetry of Fig. 1(a) the energy could be lowered, thereby giving a contradiction. 
The physics is now clear. The mathematical problem is to make rigorous sense of the lattice reflections by considering finite lattices and passing to the thermodynamic limit in an appropriate way so that at each stage the error we make is of lower order than the area $|\Lambda|$. This is accomplished by imposing periodic boundary conditions on the finite lattice, but it is much more complicated, geometrically, than a similar problem for the square lattice (with an unrealistic magnetic field flux). There, one can impose periodic boundary conditions in two orthogonal directions simultaneously. This is impossible for the hexagonal lattice since the three reflection directions are not orthogonal to each other, and we can impose periodic boundary conditions in only one direction at a time; see [16] for a discussion of this.

Nevertheless the problem can be solved and we sketch the solution here for the ground state energy. The extension to finite temperature follows by similar arguments using the reflection positivity established in $9,13-15$.

Proof sketch of the Theorem.- Step 0. The conclusion shown in Fig. 1(a) can be deduced from a statement about individual bonds. Any bond, $b$, in our lattice (except for those at the boundary) has four other bonds connected to it, as shown in Fig. 2(b). Two of these, together with $b$, form part of a hexagon and the other two, together with $b$, form part of a neighboring hexagon. We say that $b$ is happy if the first two bonds have equal $t$ values and also the second two have equal $t$-values (possibly different values). The $t$-value of $b$ itself is irrelevant for this definition. (A similar definition applies to bonds at the edge, which have fewer than four bonds attached to them.)

Our goal is to show that every bond in a minimizing configuration is happy, for this will imply the theorem. To be precise we shall show that all except $|\Lambda|^{1 / 2} \ln |\Lambda|$ of the bonds are happy, but this is good enough for the thermodynamic limit.

Step 1. We consider an increasing sequence of $\Lambda$ 's each of which is a rectangular piece of the hexagonal lattice, with even side lengths of order $L$. Approximately one third of the bonds are oriented in the $y$-direction. We periodize $\Lambda$ in the $y$-direction by connecting the sites on the top row to the corresponding sites in the bottom row to form a new row of hexagons, and call the new lattice $\tilde{\Lambda}$. This leads to a cylinder whose axis is parallel to the $x$-axis. The new hopping matrix $\tilde{T}$ consists of the old hopping matrix $T$ and some new positive elements $t_{x y}$ connecting the top to the bottom. The new Hubbard Hamiltonian $\tilde{H}(\tilde{T})$ is defined as in (1) except that the (positive) $t_{x y}$ 's which connect top to bottom are inserted with a minus sign. With this convention, every hexagon on the cylinder has flux zero and any circuit around the cylinder that encircles the cylinder axis has length $0 \mathrm{mod}$ 4 and has flux $\pi$ 18]. (The flux of a circuit is the argument of the product of the $t$ 's along this circuit.) The importance of these fluxes lies in the following fact: Re-
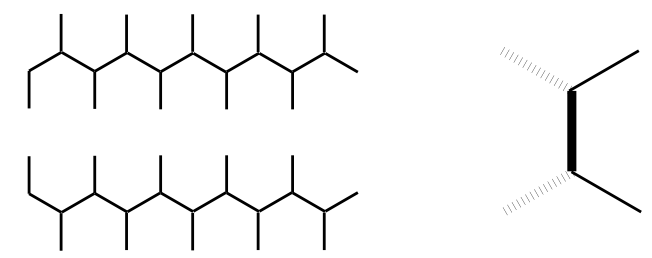

FIG. 2: Some definitions: (a) The top left figure is a single row and the bottom left figure is a reflected $\theta$ row. They are joined by overlapping the common vertical bonds. (b) A happy bond (heavy line) has the $t$-values of the four adjacent bonds equal in pairs, as shown.

flection positivity requires dividing the lattice into two pieces and this requires cutting bonds, which means cutting closed circuits. In order to have reflection positivity every closed circuit that is cut must have flux $\pi$ if the length of the circuit is $0 \bmod 4$ and flux 0 if it is $2 \bmod 4$. This is shown in [9, 17]. The length of a hexagon circuit is 6 and the length of a circuit going around the cylinder is always $0 \bmod 4$.

The insertion of additional bonds changes the energy from $E(T)$ to $\tilde{E}(\tilde{T})$, the ground state energy of $\tilde{H}(\tilde{T})$, by at most $O(L)$, i.e., order $1 / L$ per atom.

We think of this cylinder as composed of rows, parallel to the axis, as shown in Fig. 2(a), together with their reflections, which we shall call $\theta$-rows. Thus $\tilde{\Lambda}$ is a sequence of rows interspersed with $\theta$ rows. Each row and $\theta$ row has its prescribed hopping matrix elements. Let us denote the rows as we go around the cylinder by $A_{1}, \theta A_{2}$, $A_{3}, \theta A_{4}, \ldots, A_{M-1}, \theta A_{M}$ (with $M$ of order $L$ ). From these we can create $M$ new configurations $\tilde{T}_{1}, \ldots, \tilde{T}_{M}$ of matrix elements of which the $j$-th one has the rows $A_{j}, \theta A_{j}, A_{j}, \theta A_{j}, \ldots$ It is a well known consequence of reflection positivity (3) (called the chessboard estimate $[6,11,14,15]$ ) that

$$
\tilde{E}(\tilde{T}) \geq \frac{1}{M} \sum_{j=1}^{M} \tilde{E}\left(\tilde{T}_{j}\right),
$$

from which we see that one of the $\tilde{T}_{j}$ configurations is energetically at least as good as the original $\tilde{T}$ configuration. In a $\tilde{T}_{j}$ configuration every vertical bond is happy.

At this point we have established a $T$ configuration for which all the vertical bonds are happy. We have no knowledge of the happiness of the zig-zag bonds between the vertical bonds. Our next goal is to make $50 \%$ of the remaining zig-zag bonds happy without destroying the happiness of very many of the vertical bonds. This is the difficult step.

Step 2. We resist the temptation to cut the cylinder along the line by which it was originally formed. Instead, we proceed as follows, and we urge the reader to follow this construction by forming a cylinder from a piece of paper and tape. We start at one edge of the cylinder and, with a pair of scissors we cut the paper along a 

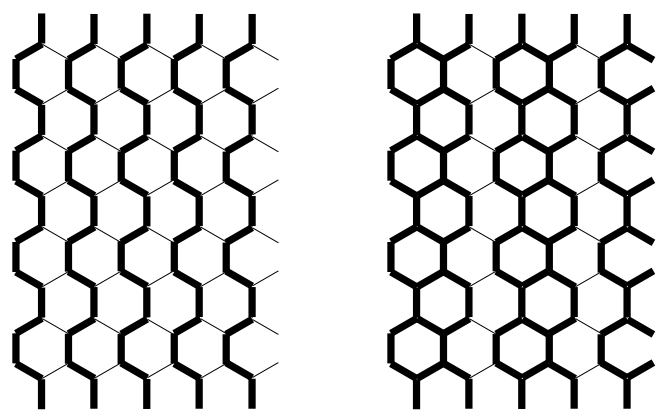

FIG. 3: (a) The heavy lines in the left figure show the more than $50 \%$ happy bonds after reflecting in orientations 1 and 2. (b) shows the more than $75 \%$ happy bonds after reflecting in orientations 1, 2 and again 1.

line that is $30^{\circ}$ from the axis (so $60^{\circ}$ from the vertical direction). The line should not go through the atoms and it should cut an even number of bonds. In this way we cut a spiral that eventually reaches the other edge of the cylinder and we lay the paper out flat on the table. It will be noted that there are two ways to tape this lozenge piece of paper together to form a cylinder. One way is just to put it back together in the way we cut it. The second way, which is the one we will use, is to connect the lozenge to itself with a shift in the $x$-direction. We pay a surface energy to accomplish this. The old vertical bonds that were parallel to the $y$-axis now lie at an angle of $60^{\circ}$ to the axis of the new cylinder (which we again assume to be parallel to the $x$-axis), and a different set of bonds now lies parallel to the $y$-axis.

Again we focus our attention on a single row of the new cylinder, as shown in Fig. 2(a). The happiness established in Step 1 means that every second zig-zag bond of the new row is happy. We now repeat the reflection positivity argument. Inequality (4) allows us to repeat one row periodically. This repetition preserves the happiness of every second bond on the zig-zag line and makes every vertical (in the new orientation) bond happy. The resulting network of happy bonds consists of chains as shown by heavy lines in Fig. 3(a).

Step 3. We repeat Step 2 by cutting a spiral and retying the cylinder as it was initially in Step 1. Since we have cut the chains from Step 2 at an angle of $30^{\circ}$ relative to the cylinder axis, we observe that every row parallel to the cylinder axis has a zig-zag line with three out of every four bonds happy. Reflection positivity (44) allows us to repeat one such row endlessly. All the resulting vertical bonds are happy and out of every sequence of four zigzag bonds three are happy. The configuration of happy bonds again consists of chains but now these chains are thicker; on every row the chain has three zig-zag bonds instead of one. This is shown in Fig. 3(b).

Step 4. We cut and paste again and use reflection positivity to achieve happiness for seven out of eight zigzag bonds. The chains are now seven bonds thick.
After $k$ steps all the vertical bonds and $2^{k}-1$ out of every consecutive $2^{k}$ zig-zag bonds are happy. We have paid a surface energy $k$ times and we also might have incorrect bonds along the sequence of cut lines, but each of these corrections can change the energy at most by a quantity of order $L$. If we take $k \sim \ln _{2} L$, we find that all except possibly order $k L$ bonds are happy, and we have paid an energy error of at most $O\left(L \ln _{2} L\right)$. This is what we wanted to show.

Conclusion. The Hubbard model for graphene on the hexagonal lattice has a special property called reflection positivity that the square lattice does not have. With the aid of this property we are able to limit the kind of lattice distortions allowed by this model. Only distortions that preserve the three-fold reflection symmetry of the lattice are possible in the thermodynamic limit.

We are indebted to G. Dunne, D. Abanin and A. Giuliani for illuminating discussions and G. Holzegel for technical help. A grant from the U.S. National Science Foundation is acknowledged: PHY-0965859 (E.L.).

* (c) 2011 by the authors. This paper may be reproduced, in its entirety, for non-commercial purposes.

† Electronic address: rlfrank@math.princeton.edu

‡ Electronic address: lieb@princeton.edu

[1] C. Hou, C. Chamon, and C. Mudry, Electron fractionalization in two-dimensional graphenelike structures, Phys. Rev. Lett. 98, 186809 (2007).

[2] A. Giuliani, V. Mastropietro, M. Porta, Lattice gauge theory model for graphene, Phys. Rev. B 82, 121418(R) (2010).

[3] J. W. Mintmire, B. I. Dunlap, C. T. White, Are Fullurene Tubules Metallic?, Phys. Rev. Lett. 68, 631 (1992).

[4] K. Okahara, K. Tanaka, H. Aoki, T. Sato, T. Yamabe, Band structures of carbon nanotubes with bondalternation patterns, Chem. Phys. Lett. 219, 462 (1994).

[5] C. Chamon, Solitons in Carbon Nanotubes, Phys. Rev. B 62, 2806 (2000).

[6] E. H. Lieb, B. Nachtergaele, The Stability of the Peierls Instability for Ring Shaped Molecules, Phys. Rev. B 51, 4777 (1995).

[7] R. E. Peierls, Quantum Theory of Solids, Clarendon Press (1955).

[8] T. Kennedy, E. H. Lieb, Proof of the Peierls Instability in One Dimension, Phys. Rev. Lett. 59, 1309 (1987).

[9] E. H. Lieb, The Flux Phase of the Half-Filled Band, Phys. Rev. Lett. 73, 2158 (1994).

[10] E. H. Lieb, Two Theorems on the Hubbard Model, Phys. Rev. Lett. 621201 (1989). Errata 62, 1927 (1989).

[11] K. Osterwalder, R. Schrader, Axioms for Euclidean Green's functions, Comm. Math. 31, 83 (1973).

[12] J. Fröhlich, B. Simon, T. Spencer, Infrared bounds, phase transitions and continuous symmetry breaking, Comm. Math. Phys. 50, 79 (1976).

[13] F. Dyson, E. H. Lieb, B. Simon, Phase Transitions in Quantum Spin Systems with Isotropic and Non-Isotropic Interactions, J. Stat. Phys. 18, 335 (1978).

[14] J. Fröhlich, E. H. Lieb, Phase transitions in anisotropic 
lattice spin systems, Comm. Math. Phys. 60, 233 (1978).

[15] J. Fröhlich, R. Israel, E. H. Lieb, B. Simon, Phase Transitions and Reflection Positivity. I. General Theory and Long Range Lattice Models, Comm. Math. Phys. 62, 1 (1978).

[16] J. Fröhlich, R. Israel, E. H. Lieb, B. Simon, Phase Transitions and Reflection Positivity. II. Lattice Systems with Short-Range and Coulomb Interactions, J. Stat. Phys.
22, 297 (1980).

[17] N. Macris, B. Nachtergaele, On the Flux phase conjecture at half-filling: an improved proof, J. Stat. Phys. 85, 745 (1996).

[18] E. H. Lieb, M. Loss, Fluxes,Laplacians and Kasteleyn's Theorem, Duke Math. Journal 71, 337 (1993). 